\title{
Preface
}

\section{Evolving Strategies in Heart Failure Management: An Eye Toward Cardiac Resynchronization Therapy}

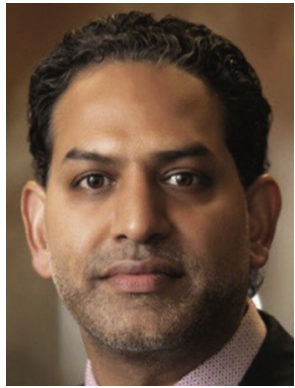

Gopi Dandamudi, MD

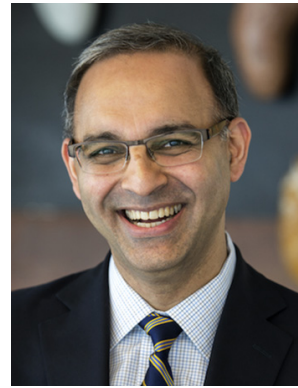

Jagmeet P. Singh, MD, PhD Editors
Over the years, the economic pressures related to access and cost of care for heart failure (HF) patients have started to strain medical practices globally. Stringent criteria, such as length of stay and 30-day readmission rates, have become ubiquitous in the medical jargon. Reimbursements have been specifically tied to such measures, resulting in significant cost pressures on health care systems. As the global population ages, the incidence of HF has continued to rise, and this has forced medical communities to concoct innovative solutions to try to curb the rising health care costs. Such innovations have included programs such as the medical home, where care has shifted from the hospital to the outpatient setting and specifically into patients' homes, and the use of multidisciplinary teams, such as social workers, health care providers, and nurse navigators, to coordinate complex care to avoid hospital admissions, reduce morbidity, and ultimately, conserve expensive medical resources.

The role of cardiac resynchronization therapy (CRT) has also evolved over the years. Earlier at- tempts had resulted in less than ideal success rates in achieving clinically meaningful ventricular synchrony and improved outcomes. Over time, with better understanding of the substrate and also with increasing investments, we have improved our success rates dramatically. With the introduction of quadripolar leads, success rates have approached $96 \%$ in some publications. Also, the role of CRT in reducing both morbidity and mortality in patients with left ventricular (LV) systolic dysfunction and wide left bundle branch block is unquestioned. However, the majority of patients with HF do not qualify for CRT therapy based on current evidence and clinical criteria.

In this issue we have had the privilege of enlisting preeminent leaders in the management of HF. The topics are aligned in a systematic fashion. We start out by describing the complexity of HF and its current toll on medical costs and contemporary treatment strategies. The role of CRT is discussed in detail along with strategies on how to achieve a successful implant in a busy clinical 
practice. A detailed discussion is also provided on how to manage patients with CRT and emphasizes the growing role of remote monitoring. The role of cardiac magnetic resonance in assessing dyssynchrony is also discussed. CRT in special populations (near preserved LV systolic function and chronic atrial fibrillation) is addressed in subsequent articles. Finally, emerging pacing technologies in HF, such as leadless pacing and permanent His bundle pacing, are highlighted at the end of the issue to inform the readers of the exciting technologies that are on the horizon.

We want to thank all the authors who have contributed with genuine enthusiasm to this issue and also the editors for granting us the opportunity to serve as guest editors. Finally, no contribution is possible without the patience and the sacrifice of our families, who continue to support our clinical and research endeavors.

Sincerely,

Gopi Dandamudi, MD Medical Director, Cardiovascular Service Line

Cardiology

$\mathrm{CHI}-$ Franciscan Health System

Tacoma, WA, USA

Jagmeet $P$. Singh, MD, PhD

Cardiology

Massachusetts General Hospital

Boston, MA 02114, USA

E-mail addresses:

gdandamu@iu.edu (G. Dandamudi)

JSINGH@mgh.harvard.edu (J.P. Singh) 